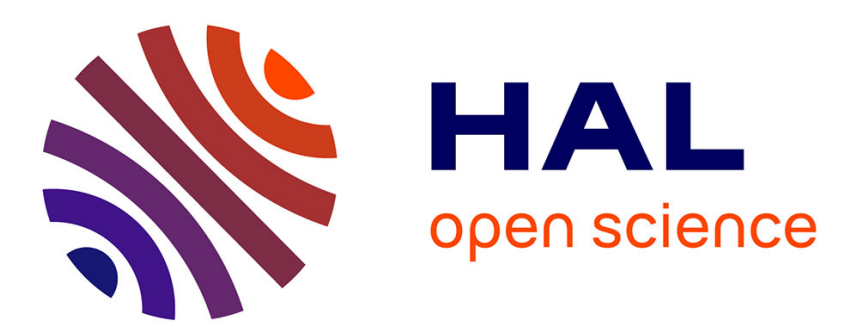

\title{
A multiscale calibration of a photon video microscope for visual servo control: Application to Micromanipulation.
}

Brahim Tamadazte, Sounkalo Dembélé, Nadine Le Fort-Piat

\section{To cite this version:}

Brahim Tamadazte, Sounkalo Dembélé, Nadine Le Fort-Piat. A multiscale calibration of a photon video microscope for visual servo control: Application to Micromanipulation.. IEEE International Workshop on Robotic and Sensors Environments, ROSE'08., Oct 2008, Ottawa, Canada. pp.29-34, 10.1109/ROSE.2208.4669176 . hal-00338541

\section{HAL Id: hal-00338541 https://hal.science/hal-00338541}

Submitted on 13 Nov 2008

HAL is a multi-disciplinary open access archive for the deposit and dissemination of scientific research documents, whether they are published or not. The documents may come from teaching and research institutions in France or abroad, or from public or private research centers.
L'archive ouverte pluridisciplinaire HAL, est destinée au dépôt et à la diffusion de documents scientifiques de niveau recherche, publiés ou non, émanant des établissements d'enseignement et de recherche français ou étrangers, des laboratoires publics ou privés. 


\title{
A Multiscale Calibration of a Photon Video Microscope for Visual Servo Control: Application to Micromanipulation
}

\author{
Brahim Tamadazte, Sounkalo Dembélé and Nadine Le Fort-Piat \\ FEMTO-ST Institute, UMR CNRS 6174 - UFC / ENSMM / UTBM, \\ Automatic Control and Micro-Mechatronic Systems Department, \\ 24 rue Alain Savary, 25000 Besançon, France \\ Phone: +33 (0)3 814027 99, Fax: +33 (0)3 81402809 , \\ E-mail: $\{$ btamadaz, sdembele, npiat\}@ens2m.fr
}

\begin{abstract}
Since many years the need of automated microassembly systems i.e. systems to assembly micrometric parts, MEMS (Micro-Electro-Mechanical Systems), MOEMS (Micro-Opto-ElectroMechanical Systems) etc., becomes more and more necessary because of the development of micro components based products. The corresponding tasks (supervision, control, quality inspection, ...) involve accurate metric measurements from images. The main image source is the photon videomicroscope whose features are the weakness of the depth-of-field and that of the field-of-view. The paper deals with the modelling and calibration of that kind of imaging system. A multiscale calibration paradigm is proposed and validated by means of a commercial video microscope. The model is derived from the usual perspective model. The latter is simplified by considering a single scale factor and then the link between the zoom and that scale factor is established. The calibration is performed through a virtual pattern made by tracking a silicon micro part in the images of the scene. The final error between the projection of a pixel and its model is about 1.45 pixel indicating the accuracy of the approach. At the end, the model is used to perform a visual servoing whose purpose is the aligning and centering of a micropart with a gripper.
\end{abstract}

Keywords - Calibration, multiscale, photon video microscope, virtual pattern, visual servoing, microassembly, micromanipulation

\section{INTRODUCTION}

Contrary to macroscopic and mesoscopic scales where assembly can be achieved without images of the scene, manipulation at microscopic scale always requires images. The manipulation of biological objects like pollens or ovocytes are typical examples of micromanipulation. But since many years the manipulation of artificial objects like monolithic microcomponents, MEMS and MOEMS, has emerged. In that case the images represent the views of the work scene from which a lot of metric informations can be derived : the pose of a component or a gripper, the distance between a component and a gripper, the speed of a gripper, ... Those data are required for the $2 \mathrm{D}$ or $3 \mathrm{D}$ reconstruction of the scene, the quality inspection or the control of manipulators.

Microassembly corresponds to assembly at the microscale, and implies the manipulation of components where the size ranges between $1 \mu \mathrm{m}$ and $1 \mathrm{~mm}$ and the accuracy about $1 \mu \mathrm{m}$ ([8]), ([6]), ([11]). As a consequence the photon videomicroscope is required as the image source in the set-up devoted to that task. The latter exhibits high resolution, but has two drawbacks which are the weakness of the field-of-view and that of the depth-of-field. For the system used in the experiments exposed below (LEICA MZ16A) the field-of-view is $700 \mu \mathrm{m} \times 900 \mu \mathrm{m}$ at the maximum of the zoom, the depth-of-field varies between $2.9 \mathrm{~mm}$ and $0.035 \mathrm{~mm}$ according to the numerical aperture of the objective. Those elements complicate the calibration and use of the video microscope.

The paper deals with that problem of calibration. Some solutions have been proposed in the literature. The main is that of Zhou and Nelson ([2]). These authors point out the differences between a standard image source (with a standard video lens) and a video microscope. They model the latter by the usual perspective non linear model in which the optical tube length $T_{o p}$ is introduced in the equations. In fact, many microscopes include a tube between the sensor and the lens (whose focal length is noticed $f$ ) in order to increase the magnification. As a consequence the field-of-view as well as the depth-of-field become weak. The parameters of their model are $T_{o p}, f, d$ and $k_{1}$ where $d$ is the distance between the object plane and the front focal plane and $k_{1}$ is the lens radial distortion. Tsai algorithm ([10]) is used to compute the above parameters and the extrinsic ones (the three Euler angles $\alpha, \beta$ and $\gamma$ ), and the translation vector $\left(T_{x}, T_{y}, 0\right)^{T}$ of a fixed zoom and focus video microscope. The calibration sample is made of squares of 10 $\mu \mathrm{m}$ side etched in a glass plate. Ammi et al. ([1]), Daflon et al. ([2]) consider the linear perspective model but use a virtual 3D pattern: a target point is tracked in the images of the scene. In the case of Ammi et al., the calibration approach of Zhang ([12]) is modified considering simplification due to microscope imaging. Figl et al. ([4]) consider the linear perspective model and the algorithm of Tsai in their experiment of calibrating a variable zoom and focus microscope. A non linear model including non paraxial distortion is introduced by Danuser ([3]) in the modelling of a stereo microscope.

In the first section of the paper the linear model of the video 
microscope is stated. It is simplified by considering a single scale factor instead of two, then the concept of multiple scale model is introduced by modelling that scale factor as a function of the magnification. That idea is similar to that developed by Tarabani et al.([9]), Li et al.([5]) and Sturm ([7]) in the case of macroscopic zoom-lens camera system. The second section describes the approach to compute the parameters of that multiple scale model using a virtual pattern made by tracking a silicon part in the work scene. The third section states the experimental results obtained with a commercial videomicroscope (MZ16A from Leica) characterized by a motorized zoom and focus. The latter is modelled and the obtained parameters are used to implement a visual servo control for the accurate positioning of a part with respect to a gripping. The paper is ended by a conclusion.

\section{MULTISCALE MODEL}

The components of any photon image source is a video lens in association with a camera. The first component focuses the rays into the surface of the sensor (of the second) which converts that photonic energy into an electric energy. According to the video lens, three types of image sources can be considered. The basic image source is the pinhole camera where no conventional lens is used. An extremely small hole in a very thin material focuses light by confining all the rays from the scene. In order to produce a reasonably clear image, the aperture has to be about a hundred times smaller than the distance to the sensor, or less.

The standard image source is more complicated than the pinhole camera, it includes a standard video lens whose focal length is greater than some millimeters. The optical magnification is very small and then the resolution is very weak. Image formation on the sensor is driven by standard geometrical and physical optics.

The last type of photon image source is the video microscope where the rays are focuses by means of a microscope. The modern compound microscope is designed to provide a magnified two-dimensional image that is focused axially in successive focal planes. It is equipped with infinity-corrected objectives which are responsible for primary image formation and play a central role in determining the quality of the image. Focal length of microscope is smaller than a millimeter. In some cases, a tube is also associated in order to increase the magnification.

\section{A. Basic Model}

Standard lens based image source is modelled by the non linear projective model. The latter includes:

- intrinsic parameters inherent to the imaging system: the focal length $\boldsymbol{f}$, the scale factors $k_{x}, k_{y}$ in $\boldsymbol{x}$ and $\mathbf{y}$ directions, the principal point coordinates $\left(x_{o}, y_{o}\right)$ (front focal point),
- extrinsic parameters corresponding to the position and orientation of the camera frame with respect to the scene frame: translation components $T_{x}, T_{y}$, and $T_{z}$, and for example Euler angles $\alpha, \beta$ and $\gamma$,

- distortion parameters: the radial $\left(a_{1}, a_{2}, \ldots\right)$ and tangential $\left(b_{1}, b_{2}, \ldots\right)$ distortion coefficients.

The improvement of technology enables the manufacturing of isotropic image sensors in which the scale factors along $x$ and $y$ are identical:

$$
k_{x}=k_{y}=k
$$

The microscope based image source is an optical imaging and then can be modelled by the non linear projective model. However, the quality of laboratory microscope is usually better than that of standard lens. Experimental values of distortion parameters are very weak: ranging from $10^{-8}$ to $10^{-10}$ ([10]), ([13]). As a consequence the distortions can be neglected, and then the video microscope can be modeled by the linear projective model.

That model consists in a perspective projection of a scene point $P$ onto a pixel $p$ in the retinal plane through the optical center.

Let $P$ and $p$ be respectively represented by the homogeneous vector $(X, Y, Z, 1)^{T}$ and $(x, y, w)^{T}$, the model is written:

$$
p=Q P
$$

The matrix $Q$, dimension $3 \times 4$, is the homogeneous projection matrix of the image source. It is written:

$$
Q=K\left[\begin{array}{llll}
1 & 0 & 0 & 0 \\
0 & 1 & 0 & 0 \\
0 & 0 & 1 & 0
\end{array}\right] * D
$$

The matrix $K$ gathers the intrinsic parameter as followes:

$$
K=\left(\begin{array}{ccc}
f k & 0 & x_{o} \\
0 & f k & y_{o} \\
0 & 0 & 1
\end{array}\right)
$$

In the microscopes where a tube is associated with the lens, the parameter $f$ in above equations becomes the sum of the focal length and the length of that tube.

The displacement matrix $D$ combines the rotation matrix [R] and the translation vector $[\mathbf{T}]$ as followed:

$$
D=\left(\begin{array}{cc}
R_{3 \times 3} & T_{1 \times 3} \\
0 & 1
\end{array}\right)
$$

If the Euler angles $\alpha, \beta$, and $\gamma$ are considered, that rotation matrix is formulated as followed:

$$
R=\left(\begin{array}{lll}
r_{11} & r_{12} & r_{13} \\
r_{21} & r_{22} & r_{23} \\
r_{31} & r_{32} & r_{33}
\end{array}\right)
$$

with: 


$$
\begin{array}{ccc}
r_{11}= & \cos \alpha \cos \beta \\
r_{12}= & \cos \alpha \sin \beta \sin \gamma-\sin \alpha \cos \gamma \\
r_{13}= & \cos \alpha \sin \beta \sin \gamma+\sin \alpha \sin \gamma \\
r_{21}= & \sin \alpha \cos \beta \\
r_{22}= & \sin \alpha \sin \beta \sin \gamma+\cos \alpha \cos \gamma \\
r_{32}= & \sin \alpha \sin \beta \cos \gamma-\cos \alpha \sin \gamma \\
r_{13}= & -\sin \beta \\
r_{32}= & \cos \beta \sin \gamma \\
r_{33}= & \cos \beta \cos \gamma
\end{array}
$$

\section{B. Multiscale Model}

Multiscale calibration is required because the video microscope considered in the experiments, works at multiple zoom or magnification. Then it is necessary to introduce the zoom factor $\zeta$ in above intrinsic model. The solution we adopt is to consider only the scale factor $k$ as a non linear function of the zoom factor $\zeta(k(\zeta))$. It is assumed that the others parameters are not modified by the zoom factor. The model becomes:

$$
K(\zeta)=\left(\begin{array}{ccc}
f k(\zeta) & 0 & x_{o} \\
0 & f k(\zeta) & y_{o} \\
0 & 0 & 1
\end{array}\right)
$$

As a consequence the knowledge of the zoom factor $\zeta$ enables the estimation of the focal length as followed:

$$
f=\frac{K_{11}(\zeta)}{k(\zeta)}
$$
$K$.

with $K_{11}=f k$, the first element of the calibration matrix

\section{Application in Visual Servo Control}

The purpose of the above calibration scheme is the implementation of $2 \mathrm{D}$ visual servoing to perform some tasks of robotic manipulation, especially the aligning and centering of a micropart with respect to a gripper. Those tasks require high accuracy that can be obtained through $2 \mathrm{D}$ visual servoing known to be precise and robust to the errors of modelling of the robotic and imaging systems.

Multiscale calibration leads to multiscale visual control. The desired value of any visual feature point $(s *)$ and the current value of that point $(s)$ become functions of the zoom factor $\zeta$. As a consequence the interaction matrix $L_{s}$ integrates that zoom factor, then the usual visual servo control scheme is modified as represented in (Fig.1) where $L_{s}$ and $\lambda_{a}$ are respectively the pseudo-inverse of the interaction matrix $L_{s}$ and the gain of decrease. The change of the zoom causes the change of the control parameters.

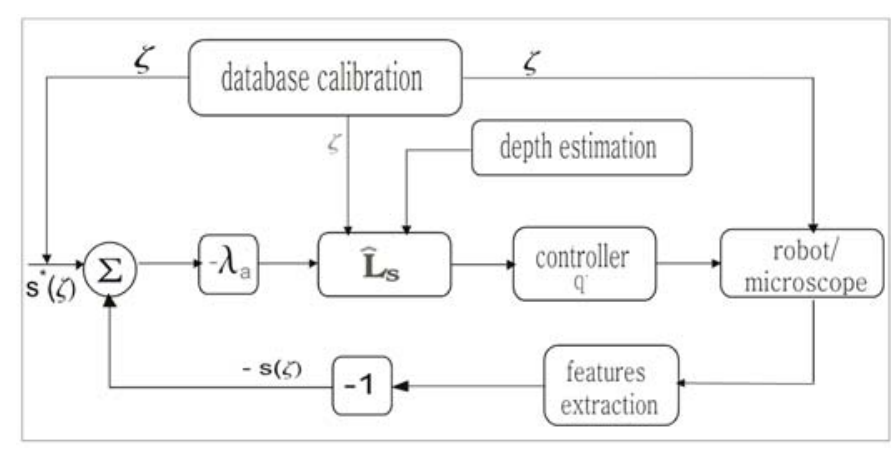

Fig. 1.The functional chart of multiscale visual servoing

\section{COMPUTING THE PARAMETERS OF THE MODEL}

There are similarities between vision sensing in the macro, meso and micro scales. In every scale a 2D image is formed from a 3D object through an optical lens system. As they are modelled by the same model, the same approaches of calibration can be performed. Those techniques are roughly classified into two families: the photogrammetric calibration approaches and the self calibration approaches.

- In photogrammetric approaches, the model parameters are computed by observing a calibration object (3D pattern) whose geometry is known with very high accuracy. The calibration object usually consists of 2 or 3 planes orthogonal to each other. A unique plane object is also used, but in this case several images at different 3D orientations should be considered ([10]).

- Self calibration approaches do not necessarily use a calibration pattern, the correspondences between some images of a static scene are sufficient to recover both the intrinsic and extrinsic parameters. In that case the accuracy is less better than in the approaches described above ([13]).

In any case an important number of correspondences $p-P$ are required. The calibration is performed in two stages.

- The first stage consists in estimating roughly the projection matrix $Q$ by resolving the DLT obtained by combining the projection equations for the point correspondences.

- The second stage enables the fine estimation of the parameters by minimizing a criterium, usually the geometric error:

$$
\sum_{i} d\left(p_{i}, Q P_{i}\right)
$$

Even if the same algorithm can be implemented both in macro and micro scales, they are some fundamental differences between standard lens and microscope based image sources calibration. A microscope is characterized by very weak depth-of-field and field-of-view. For the system used in the experiments exposed below, the field-of-view is $700 \mu \mathrm{m}$ $\times 900 \mu \mathrm{m}$ at the maximum zoom and the depth-of-view is 


\section{$0.035 \mu \mathrm{m}$.}

As a consequence of those limitations, a 3D calibration sample cannot be used, the sample is reduced to a planar object that should be almost parallel to the lens. A video microscope is heavy and cumbersome and then is not easy to manipulate. Those constraints complicate the calibration. On the other hand, the presence in the set-up of accurate motion sources as XYZ stages enables the performing of accurate motions of the plane calibration sample and contributes to facilitate the calibration.

Instead of using a real sample or virtual points as encountered in the literature, the proposition is to used the power of image processing: the center of a micrometric part is tracked in the images of the scene. The part is moved accurately by a $\mathrm{XYZ}$ stage, so its center position in the scene is known with high accuracy. Those positions are used to synthesize a virtual image which is used in the algorithm exposed above. That approach enables high accuracy in the computing of the model parameters. The approach is also simple, since it does not require the micromachining of any pattern. The components to assembly are directly used.

\section{EXPERIMENTS}

Above propositions are validated using a set-up including a multiple scale imaging system (a stereo video microscope MZ16A from Leica), a robotic system and a gripping system. All the elements are placed in a clean room in order to control the temperature and humidity of the environment.

\section{A. The Robotic and gripping Systems}

They include the following elements:

- a 3 DOF positioning platform (2 linear stages ( $x$ and $y$ ), and 1 rotating stage $(\theta)$ ),

- a 2 DOF micromanipulator (1 vertical linear stage $(z)$ and 1 rotating stage mounted at 45 degrees from the vertical one),

- an end-effector (microgripper) mounted on the micromanipulator (it is 4 DOF system).

\section{B. The Imaging System}

It includes:

- a video stereomicroscope (of the type LEICA MZ 16 A from Leica) for the top of view.

- a video microscope (based on long tube) for the side view.

The second image source is not considered in the paper.

The particularity of the MZ $16 \mathrm{~A}$ is the fact that the change of the zoom (magnification) is achieved by the optical block translating along $Z$ axis. The zoom does not modify the focal length since all the objective system remains invariant. Then, zooming followed by focusing do not change the intrinsic parameters, the only modification occurs on the component $T_{z}$ of the translation vector [T] of the extrinsic parameters.

The depth-of-field of the MZ 16 A varies between $2.9 \mathrm{~mm}$ for the smallest zoom factor to $0.035 \mathrm{~mm}$ for the greatest zoom factor. Then, sharp images can be obtained only when the object is located in a very narrow region around the focused plane. Those limitations justify the performing of above calibration concept.

\section{Autofocus Achievement}

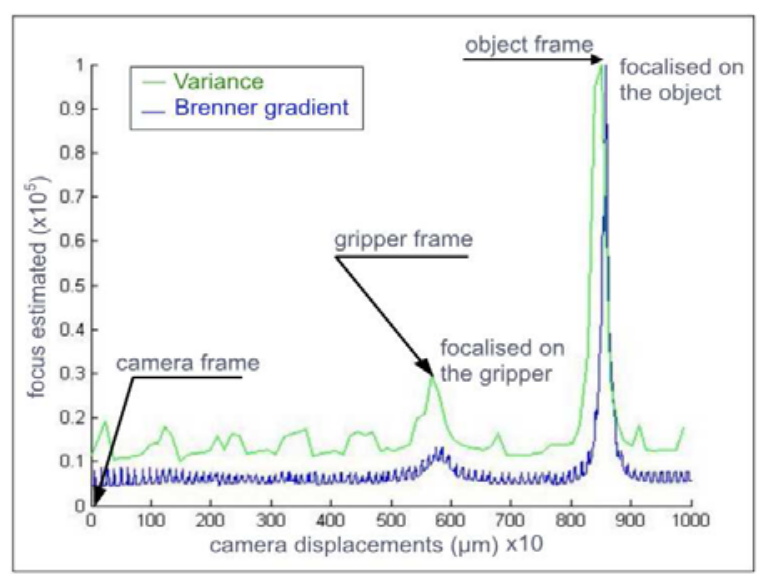

Fig. 2. The focus estimation according to the position of the microscope

Because of the weakness of the depth-of-field as exposed above, an autofocus method is implemented in order to guarantee the obtaining of focused images during the experiments. The depth of the scene is scanned step by step, images are acquired and the focus is estimated. The system is repositioned in the position where the focus estimation is the highest. The variance $F_{v a r}$ is used as the focus estimator:

$$
F_{\mathrm{var}}=\frac{1}{H \cdot W \cdot \mu} \sum_{H} \sum_{W}(i(x, y)-\mu)^{2}
$$

with:

- $H$ and $W$ respectively the image height and width

- $\mu$ the image intensity average.

Fig.2 shows the evolution of the focus according to the position of the microscope. The sharpness of the peak indicates the relevance of the method.

\section{The Scale Factor Calibration}

The following calibration sample is used: 3 holes are engraved in a metal part. The diameters of the holes are estimated 
to $1961.5,1037.5$ and $429.85 \mu \mathrm{m}$ using a SEM of $10 \mathrm{~nm}$ resolution. According to the zoom value the relevant hole image is considered in the computation of the scale factor.

The zoom factor $\zeta$ is modified with a step of 0.5 and 172 images are acquired. In every image the relevant hole is detected using a normalized correlation and the scale factor $k(\zeta)$ corresponding to the ratio of the diameter of the hole in $\mu \mathrm{m}$ $\left(S_{s}\right)$ by it diameter in pixels $\left(S_{p}(\zeta)\right)$ is computed:

$$
k(\zeta)=\frac{S_{s}}{S_{p}(\zeta)}
$$

Fig. 3 represents the evolution of the scale factor $k(\zeta)$ according to the zoom factor (or magnification) $\zeta$. The approximation of that function by a polynomial using MatLab gives a polynomial $P$ of degree ten:

$$
P=\left[p_{1} p_{2} \ldots p_{11}\right]
$$

ordered by increasing powers of $\zeta$.

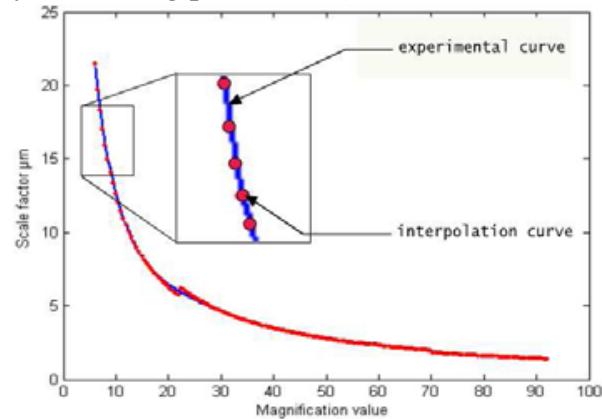

Fig. 3. The representation of the scale factor according to the magnification value

\section{E. The Virtual Pattern Construction}

The object used to achieve the virtual pattern is a silicon micro part of dimensions $400 \mu \mathrm{m} \times 400 \mu \mathrm{m} \times 100 \mu \mathrm{m}$ (Fig.4).

Steps of displacement of $300 \mu \mathrm{m}$ in $\mathbf{x}$ and $\mathbf{y}$ directions are performed, and the virtual pattern is generated as exposed above (Fig.4). Some irregularities are noticed corresponding to the geometry of the scene.
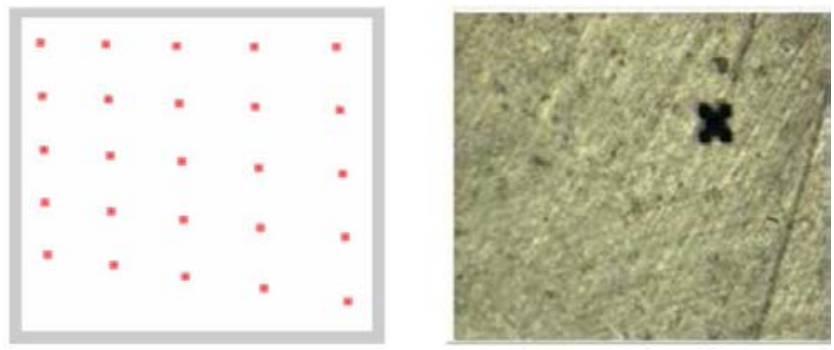

Fig. 4. The automatic tracking of the part and the virtual pattern obtained

\section{F. The Model at a Fixed Zoom Factor}

For a zoom position of $38 \times$, the computation of the calibration matrix gives:

- $K=\left[\begin{array}{ccc}3146.3 & 0 & 512.5 \\ 0 & 3146.3 & 385.5 \\ 0 & 0 & 1\end{array}\right]$ (pixels)

According to the equations, the following values are derived:

- $k=k_{x}=k_{y}=3.6444 \mu \mathrm{m}$

- $f=11.466 \times 10^{3} \mu \mathrm{m}$

And the extrinsic parameters are:

- $\alpha=88.73^{\circ}, \beta=-27.26^{\circ}$ and $\gamma=4.01^{\circ}$

- $T_{v}=[822,963,13156]^{T}(\mathrm{~cm})$

\section{G. Calibrating with a Real Sample}

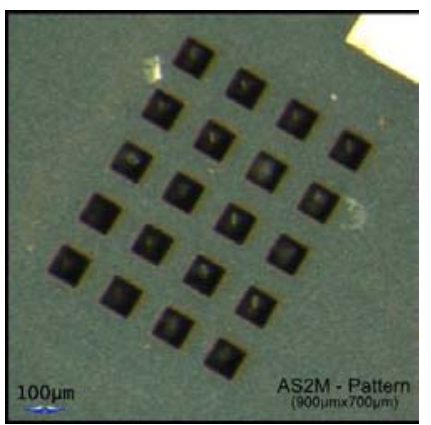

Fig. 5. The real pattern

Previous parameters are estimated using a real calibration sample: 20 square holes of $100 \mu \mathrm{m}$ side and $380 \mu \mathrm{m}$ depth are etched in a silicon wafer (Fig.5). The same algorithm is used for the extrinsic and intrinsic parameters estimation. The zoom factor is $\zeta=38 \times$, as for the model estimation using the virtual pattern. The results obtained are very close to that of the first approach. However, the mean error has doubled, i.e. $E_{m}=$ $3.1446 \mu \mathrm{m}$. The calibration accuracy depends on the engraving precision. On another side, the results are strongly dependent of the image quality obtained by the microscope. The use of the real pattern appears to be not practical in the micromanipulation case. It is very difficult to install and remove the calibration target in the confined space of manipulation under the optical microscope.

\section{H. Application to Visual Servo Control}

As exposed above the calibration parameters are used to perform the task of aligning and centering a part with respect to a gripper by visual servo control. Fig. 6 shows some shots during the achieving of those tasks, the final error is 0.5 degree and $2.6 \mu \mathrm{m}$ showing the relevance of the approach. 

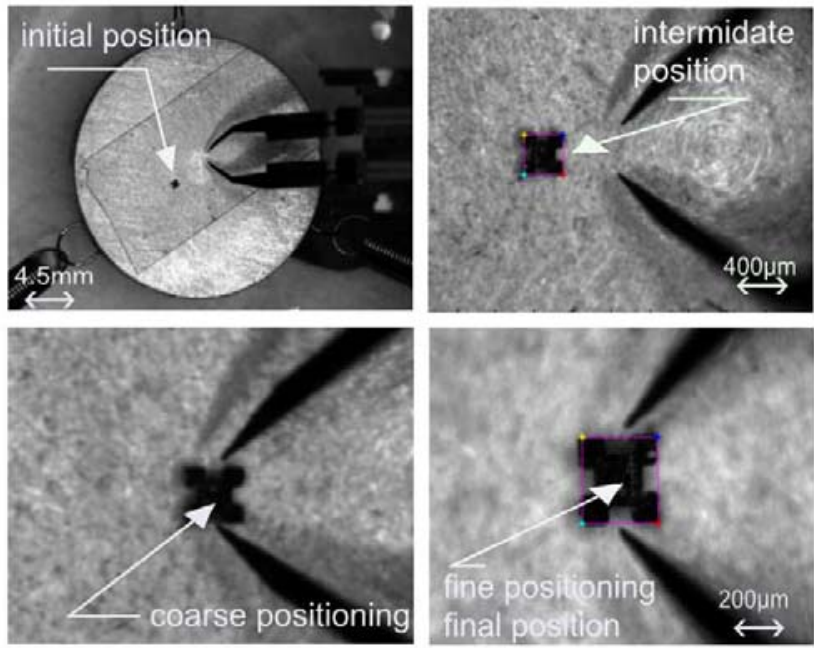

Fig. 6. Some shots during the alignment and centering of the part with respect to the gripper

\section{CONCLUSION}

Robotic microassembly corresponds to the assembly of micrometric (i.e. submillimeter) parts, to get compound products, by means of a robotic system in conjunction with a gripping system and an imaging system. The latter delivers images from which a lot of informations can be extracted for multiple use (surveillance, control, quality inspection, ...). A difficult aspect of the problem is the simultaneous requirement of high resolution information with low resolution information, the formers give accurate view of the scene when the latter give global one. The interesting solution to that multiscale imaging is the photon video microscope with electronically variable zoom.

The paper deals with the multiscale modelling of the variable zoom photon video microscope. It is shown that the functioning of the latter can reasonably be described by a simplified projective model. The first simplification is relative to the omission of distortions because of the weak values of their coefficients. The second simplification consists in considering equal the scale factor along the axis $x$ and $y$. Finally the (intrinsic) parameters become the focal length $f$, the scale factor $k$, the principal point coordinates $\left(x_{0}, y_{0}\right)$. The concept of multiple scale modelling is introduced through the zoom factor $\zeta$ whose mathematical relation with the scale factor $k$ is claimed: $k$ is a polynomial of $\zeta(k=k(\zeta))$.

Then the following approach of calibration is proposed: the polynomial $k(\zeta)$ is accurately established by changing the zoom factor $\zeta$ and computing the corresponding scale factor $k$, and the linear projective model is computed using a usual calibration approach but with a virtual calibration sample. The latter is obtained by mapping some successive centers of a micrometric part in the scene with their correspondences in the image.
The concepts are tested using a set-up including a robotic system with accurate positioning stages, a photon video microscope (MZ16A from Leica) and a silicon micropart (400 $\mu \mathrm{m} \times 400 \mu \mathrm{m} \times 100 \mu \mathrm{m})$. The polynomial is of degree ten and the focal length is $11.5 \mathrm{~mm}$. The final error of calibration is about $1.45 \mu \mathrm{m}$ indicating the rightness of the approach. These results are then used to implement a visual servo for the task of aligning and centering a part with respect to a gripper. The following errors are obtained, 0.5 degree for the orientation and $2.6 \mu \mathrm{m}$ for the position. They show the relevant of developed concepts.

\section{AKNOWLEDGEMENTS}

This work is conducted with financial support from the project Hybrid Ultra Precision Manufacturing Process Based on Positional and Self assembly for Complex Micro-Products (HYDROMEL NMP2-CT-2006-026622) funded by the European Commission.

\section{REFERENCES}

[1] M. Ammi, V. Fremont, and A. Ferreira, Flexible microscope calibration using virtual pattern for 3-d telemicromanipulation, IEEE ICRA (2005).

[2] M. Daflon, B. Lorent, and R. Clavel, A micromanipulation setup for comparative tests of microgrippers, International Symposium on Robotics, Germany (2006).

[3] G. Danuser, Stero light microscope calibration for $3 d$ submicron vision, Int. Archives of Photogrammetry and Remote Sensing, 1996.

[4] M. Figl, C. Ede, J. Hummel, F. Wanschitz, R. Ewers, H. Bergmann, and W. Birkfellner, A fully automated calibration method for an optical seethrough head-mounted operating microscope with variable zoom and focus, IEEE Tran. on Medical Imaging Vol. 24, N 11 (2005), 1492-1499.

[5] M. Li and J.M. Lavest, Some aspects of zoom-lens camera calibration, IEEE Trans. on Pattern Analysis and Machine Intelligence 18 (11) (1996), pp. 1105-1110.

[6] S. Ralis, B. Vikramaditya, and B. J. Nelson, Micropositioning of a weakly calibrated microassembly system using coarse-to-fine visual servoing strategies, IEEE Trans. on Electronics Packaging Manufacturing Vol. 23 (2) (2000), 123-131.

[7] P. Sturn, self-calibration of a moving zoom-lens camera by precalibration, IVC 15 (08) (1997), pp. 583-589.

[8] W.T. Sun and T.C. Chin, Image-based visual servo for micromanipulation : a multiple-view and multiple-scale approach, International Symposium on Micro-Nanomechatronics and Human Science, 2004.

[9] K. Tarabani, R.Y. Tsai, and D.S. Goodman, Calibration of a computer controlled robotic vision sensor with zoom lens, CVGIP (59) (1994).

[10] R.Y. Tsai, A versatile camera calibration techniaue for high-accuracy $3 d$ machine vision metrology using off-the-shelf tv cameras and lenses, IEEE Journal of Robotics and Automation Vol. RA-3, N 4 (1987), 323344.

[11] G. Yang, J. Gaines, and B.J. Nelson, Optomechatronic design of microassembly systems for manufacturing hybrid microsystems, IEEE Trans. on Industrial Electronics 52 (2005), no. 4, 1013-1023.

[12] Zhengyou Zhang, Flexible camera calibration by viewing a plane from unknown orientations, IEEE International Conference on Computer Vision, 1999, 666-673, 1999.

[13] Y. Zhou and B.J. Nelson, Calibration of a parametric model of an optical microscope, Optical Engeenering Vol. 38, N 12 (1999), pp.1989-1995. 